(1)

CrossMark

\title{
New method for rapid and dynamic quantification of elastase activity on sputum neutrophils from patients with cystic fibrosis using flow cytometry
}

\author{
Matthias Hagner (10 1 2,3,10, Dario L. Frey $\mathbb{1}^{1,2,3,10}$, Matteo Guerra $2,3,4,5,10$, \\ A. Susanne Dittrich ${ }^{1,2,3,6}$, Victoria S. Halls ${ }^{7}$, Sabine Wege ${ }^{2,3,6}$, \\ Felix J.F. Herth $2,3,6$, Carsten Schultz $2,3,4,7,11$ and Marcus A. Mall (1) 1,2,3,8,9,11
}

Affiliations: ${ }^{1}$ Dept of Translational Pulmonology, University of Heidelberg, Heidelberg, Germany. ${ }^{2}$ Translational Lung Research Center (TLRC), Heidelberg, Germany. ${ }^{3}$ German Center for Lung Research (DZL), Germany. "Molecular Medicine Partnership Unit (MMPU), European Molecular Biology Laboratory, Heidelberg, Germany. ${ }^{5}$ EMBL and Heidelberg University, Faculty of Biosciences, Heidelberg, Germany. ${ }^{6}$ Dept of Pulmonology and Critical Care Medicine, Thoraxklinik at the University of Heidelberg, Heidelberg, Germany. ${ }^{7}$ Dept of Chemical Physiology and Biochemistry, Oregon Health and Science University, Portland, OR, USA. ${ }^{8}$ Dept of Pediatric Pulmonology, Immunology and Critical Care Medicine, Charité - Universitätsmedizin Berlin, Berlin, Germany. ${ }^{9}$ Berlin Institute of Health, Berlin, Germany. ${ }^{10}$ Equal contribution. ${ }^{11}$ Equal contribution as senior author.

Correspondence: Marcus A. Mall, Dept of Pediatric Pulmonology, Immunology and Critical Care Medicine, Charité - Universitätsmedizin Berlin and Berlin Institute of Health, Augustenburger Platz 1, 13353 Berlin, Germany. E-mail: marcus.mall@charite.de

@ERSpublications

Small molecule FRET flow cytometry is a new method that enables rapid and sensitive quantification of surface-bound elastase activity on sputum neutrophils from patients with cystic fibrosis and potentially other neutrophilic airway diseases http://bit.ly/2IegeSB

Cite this article as: Hagner M, Frey DL, Guerra M, et al. New method for rapid and dynamic quantification of elastase activity on sputum neutrophils from patients with cystic fibrosis using flow cytometry. Eur Respir J 2020; 55: 1902355 [https://doi.org/10.1183/13993003.02355-2019].

\section{Introduction}

Increased activity of the serine protease neutrophil elastase (NE), secreted by activated neutrophils in the airways, is a key risk factor for the onset and progression of structural lung damage and lung function decline in patients with cystic fibrosis (CF) and non-CF bronchiectasis [1-6]. In addition to progressive structural lung damage, increased NE activity has been implicated in mucus hypersecretion [7-9], perpetuation of airway inflammation [10], and impaired host defence against Pseudomonas aeruginosa infection [6,11-13]. These studies suggest increased NE activity in sputum or bronchoalveolar lavage fluid (BALF) as a promising biomarker of airway inflammation in CF and potentially other neutrophilic airway diseases $[2-4,6]$.

To date, measurements of NE activity have focused on its free activity that becomes detectable in BALF or sputum supernatant once the overall elastase burden exceeds the inhibitory capacity of endogenous anti-proteases such as $\alpha 1$-antitrypsin and secretory leukocyte protease inhibitor [6]. After this anti-protease shield is broken, free NE activity can be measured by various methods ranging from activity-based immunoassays [5] to fluorogenic substrate-based kinetic assays [1, 2, 5] and Förster resonance energy transfer (FRET) reporter-based assays [14-16]. 
Why measure elastase activity on the surface of airway neutrophils?

$\mathrm{NE}$ is a highly cationic protein resulting in binding of a substantial proportion of secreted NE to the neutrophil surface via electrostatic interactions [17, 18]. Previous experimental work showed that this surface-bound fraction of NE confers proteolytic activity and is resistant to inhibition by endogenous anti-proteases [17, 19, 20]. Recent observational studies demonstrated that increased exocytosis and surface-bound activity of NE are already present in young children with CF and mice with CF-like lung disease, long before free NE activity becomes detectable [14, 21]. Furthermore, it was found that surface-bound NE activity correlates with early lung damage in children and severity of lung disease in adult patients with CF independent of free NE activity $[14,22]$. These results support an important role of surface-bound NE activity in pathogenesis and as a potential novel biomarker of CF lung disease across a broad spectrum of patient age and disease severity.

\section{How can elastase activity be measured on the surface of airway neutrophils?}

For measurements of surface-bound NE activity on airway neutrophils, we previously developed a lipidated ratiometric FRET reporter that inserts into the plasma membrane and contains a peptide sequence that is recognised and cleaved by NE $[23,24]$. In addition, we demonstrated that this lipidated FRET reporter enables sensitive and specific measurement of surface-bound NE activity via change in FRET signal measured by confocal microscopy (figure la and b). However, widespread implementation of this method is hampered by the time required to process and prepare the samples and acquire and analyse images (the whole procedure takes up to $8 \mathrm{~h}$ ), and by the need for expensive high-end microscopy equipment. Here, we present a new technique based on the combination of a lipidated FRET reporter and multi-colour flow cytometry, called small molecule (sm) FRET flow cytometry [25]. This technique enables quantitative assessment of NE activity on cell surfaces in a fast, robust and dynamic fashion.

\section{How does the measurement of surface-bound elastase activity by flow cytometry work?}

The lipidated FRET reporter relies on two fluorescent dyes: an energy donor (Coumarin 343) and an energy acceptor (5/6-TAMRA). The donor dye is connected to an aliphatic chain serving as a lipid anchor via a lysine residue and an amino acid sequence that is a substrate for NE. Two short polyethylene glycol spacers flank and connect the amino acid sequence with the acceptor and donor dyes (figure 1a) [23]. The close $(<10 \mathrm{~nm})$ proximity of the energy donor and acceptor allows FRET to occur efficiently when coumarin is excited. Similar to fluorophore-conjugated antibodies routinely employed in flow cytometry, this lipidated FRET reporter localises to the cell surface, where the hydrophobic moiety tethers the molecule to membrane lipids, while negative charges prevent its premature internalisation [23]. When active NE is present at the neutrophil surface, it cleaves the target peptide sequence of the FRET reporter resulting in the diffusion of the acceptor fluorophore away from the cell membrane, thereby impeding FRET (figure 1a and b). To advance FRET-based measurements of surface-bound NE activity from confocal microscopy to flow cytometry, we designed a panel of fluorochrome-conjugated antibodies consisting of anti-CD14 (monocytes, Pe/Cy7, BD Bioscience, 557742), CD16 (neutrophils, AF700, BD Bioscience, 557 920), CD45 (leucocytes, APC-Cy7, BD Bioscience, 557833 ), and CD66b (granulocytes, Pe/ Dazzle594, BioLegend, 305122 ) to selectively gate airway neutrophils as $7-\mathrm{AAD}^{\text {neg }} / \mathrm{CD} 45^{\text {pos }} / \mathrm{CD} 14^{\text {neg }} /$ $\mathrm{CD} 16^{\text {pos }}$ and $\mathrm{CD} 6 \mathrm{~b}^{\text {pos }}$ cells (figure 1c). In addition, we stained for CD63 (AF647, BioLegend, 353016 ) as a marker for primary granule exocytosis. Spectral compensation and fluorescence minus one controls were performed for all antibodies and the live dead marker. Sputum cells were isolated as previously described [22], filtered through a $40 \mu \mathrm{m}$ strainer to remove mucus and avoid clogging of the flow cytometer and stained with this antibody panel, as well as a viability marker (7-AAD). Cell viability in all samples was $>70 \%$. An aliquot of stained cells was incubated for $10 \mathrm{~min}$ at room temperature with the NE inhibitor sivelestat (CAS number 201677-61-4; Cayman Chemical, Ann Arbor, MI, USA) at a concentration of $225 \mu \mathrm{mol} \cdot \mathrm{L}^{-1}$ to inhibit reporter cleavage, and these samples served as negative controls [22, 26]. Subsequently, the lipidated NE FRET reporter (NEmo-2) was added and surface-bound activity was measured by flow cytometry at the indicated time points (figure $1 \mathrm{~d}$ and $\mathrm{h}$ ). In our setup, the flow cytometer was equipped with three lasers emitting at $405 \mathrm{~nm}, 488 \mathrm{~nm}$, and $633 \mathrm{~nm}$, respectively. For the detection of protease activity, the reporter was excited with the $405 \mathrm{~nm}$ laser. The donor signal was detected between $425 \mathrm{~nm}$ and $475 \mathrm{~nm}(450 / 50 \mathrm{~nm}$ filter) and the acceptor signal between $564 \mathrm{~nm}$ and $606 \mathrm{~nm}(585 / 42 \mathrm{~nm}$ filter) with an upstream longpass filter $(550 \mathrm{~nm})$. The FRET ratio was calculated by dividing the donor and acceptor mean fluorescence intensity values measured on the gated neutrophil population at given time points. Then, the donor/acceptor ratio was normalised to the $t=0$ donor/acceptor ratio of the negative control to ensure intra- and inter-assay stability. A change in FRET ratio represents a semi-quantitative read-out of enzymatic activity. The optimal time point for the analysis of activity is 
a)

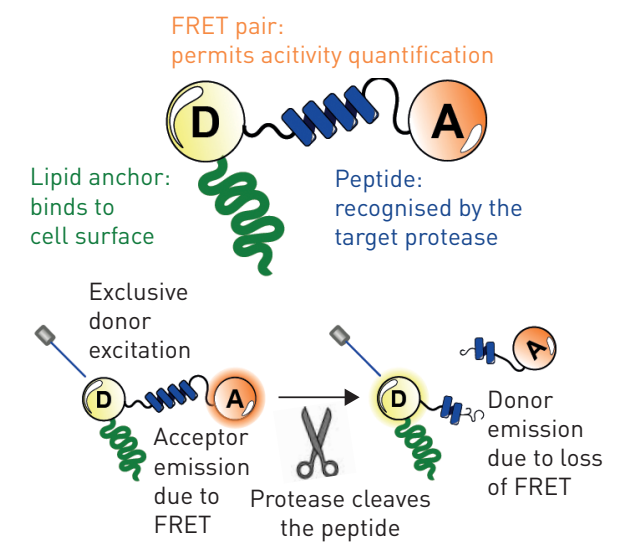

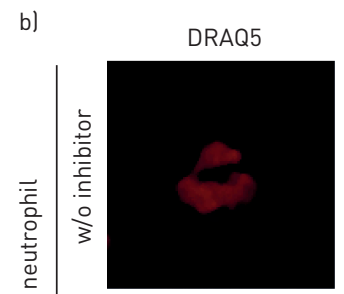
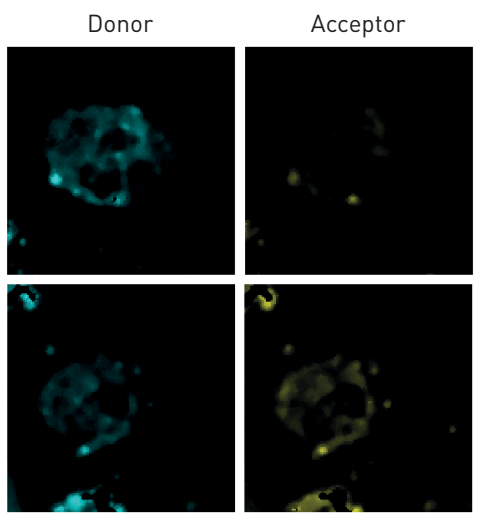

D/A ratio

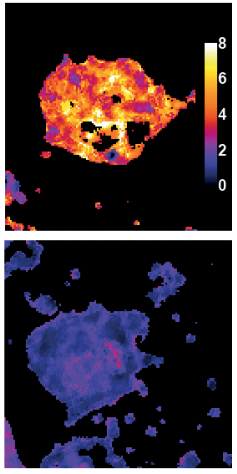

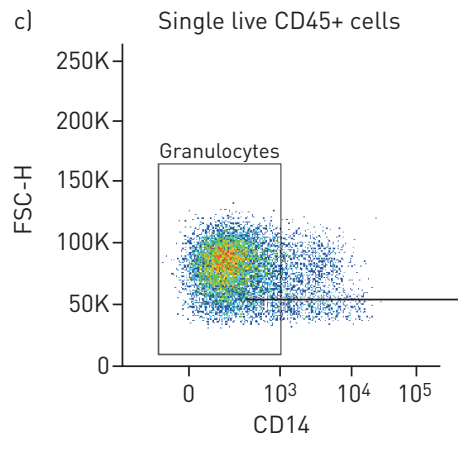

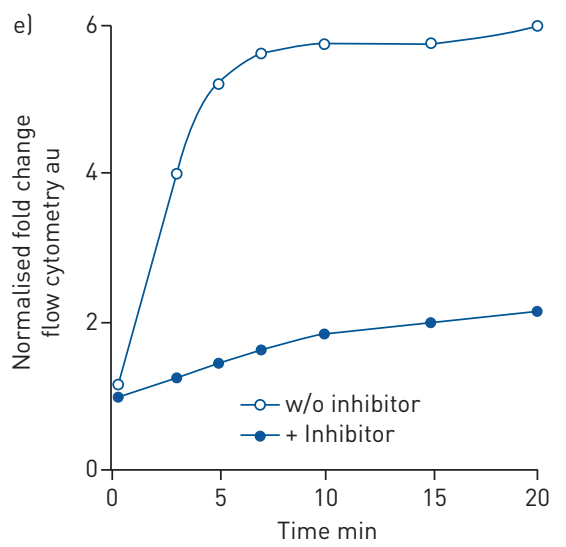

h)
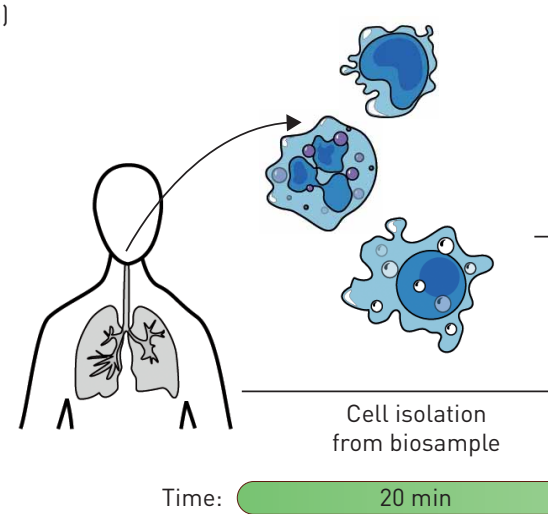
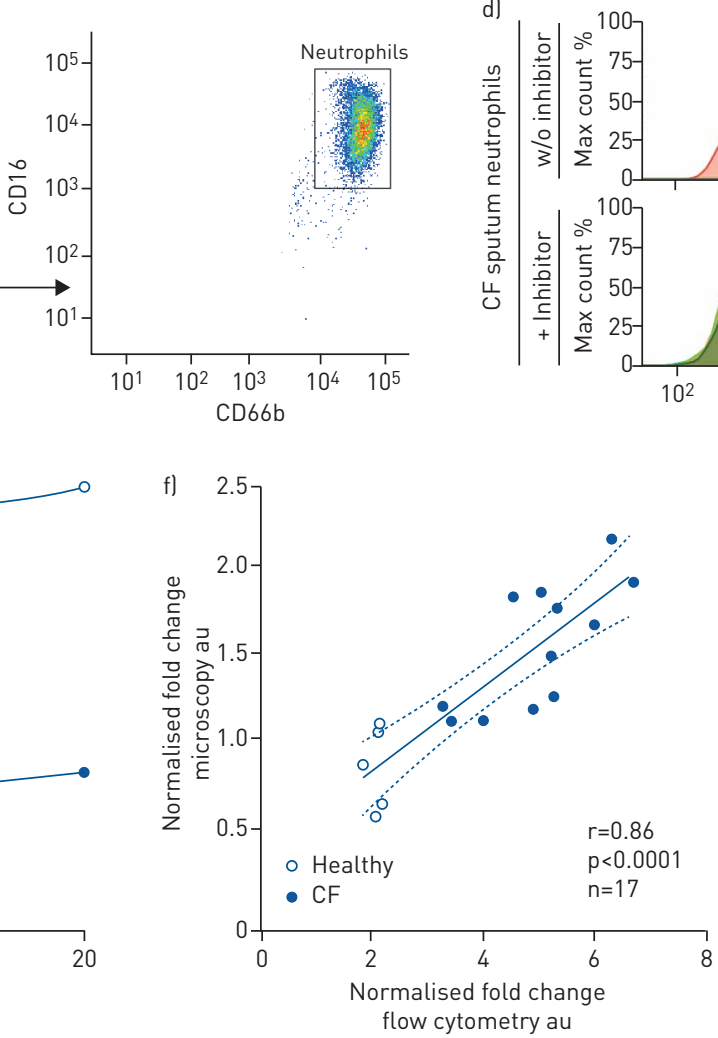


flow cytometry au


$1 \mathrm{~min}$

$+$

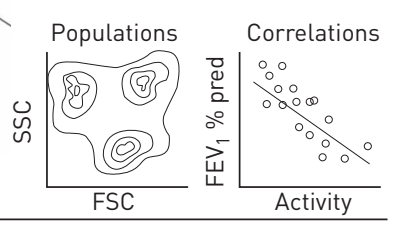

Flow cytometry analysis and statistics

$30 \mathrm{~min}$

$10 \mathrm{~min}$

$=\sim 1 \mathrm{~h}$ 
FIGURE 1 Rapid quantification of elastase activity on sputum neutrophils from patients with cystic fibrosis (CF) using a lipidated small molecule (sm) FRET reporter in combination with flow cytometry. a) Structure and mechanism of action of smFRET reporters. b) Confocal microscopy images of CF sputum neutrophils showing donor/acceptor (D/A) intensities after 10 min of incubation with the surface-bound neutrophil elastase (NE) FRET reporter (NEmo-2) without (w/o) and with (+) the NE inhibitor sivelestat. c) Gating strategy to identify sputum neutrophils in smFRET flow cytometry. d) Donor and acceptor mean fluorescence intensities of NEmo-2 measured in the absence (upper panels) and presence (lower panels) of NE inhibitor by flow cytometry on $\sim 1000$ sputum neutrophils of a patient with CF. e) Donor/acceptor ratio of NEmo-2 determined by flow cytometry over a period of $20 \mathrm{~min}$. Mean fluorescence intensities of donor and acceptor were derived from 1000 CF sputum neutrophils in the absence and presence of the NE inhibitor. Donor/acceptor ratio was normalised to the inhibited negative control donor/acceptor ratio at $t=0$ min. f) Correlation between flow cytometry and confocal microscopy measurements of surface-bound $N E$ activity at $t=10$ min on sputum neutrophils from healthy individuals and patients with CF. g) Correlation between surface-bound NE activity measured by smFRET flow cytometry and forced expiratory volume in $1 \mathrm{~s}\left(\mathrm{FEV}_{1}\right) \%$ predicted in patients with CF. Correlation analyses were performed using the Spearman rank order method. A p-value $<0.05$ was considered statistically significant. h) Experimental workflow of smFRET flow cytometry. Sputum cells are isolated from healthy and diseased subjects. Cells are stained with an adequate antibody panel that allows the identification of the cell population of interest. After antibody staining, the reporter is added to the cells, the sample is subsequently analysed by flow cytometry, surface-bound protease activity is determined, and results are available within approximately $1 \mathrm{~h}$.

within the plateau phase of the donor/acceptor ratio which we identified for our experimental conditions to be at $\mathrm{t}=10 \mathrm{~min}$ (figure $1 \mathrm{e}$ ).

To validate this new method, we analysed surface-bound NE activity with NEmo-2 on sputum neutrophils from 12 patients with $\mathrm{CF}$ (mean \pm SD age $30.9 \pm 11.8$ years, range 22.1-58.2 years) and five healthy controls (mean \pm SD age $36.6 \pm 10.0$ years, range 27.1-49.1 years). CF patients had the following CFTR genotypes: F508del/F508del ( $n=6)$, F508del/I507del $(n=1)$, F508del/CFTR dele 17 (2.5 kb) (n=1), F508del/T1299I $(\mathrm{n}=1)$, R553X/M1101K $(\mathrm{n}=1), \mathrm{W} 1282 \mathrm{X} / 2991 \mathrm{del} 32(\mathrm{n}=1)$, and G542X/X $(\mathrm{n}=1)$. Seven patients were chronically colonised with Pseudomonas aeruginosa, eight with Staphylococcus aureus and three with Aspergillus fumigatus. At the time of sputum collection, 10 patients were clinically stable and on inhaled or oral antibiotics as standard of care, and two patients had a pulmonary exacerbation and were treated with i.v. antibiotics. We performed side-by-side measurements using confocal microscopy and the new smFRET flow cytometry on sputum neutrophils from all 17 subjects. All analyses either performed by confocal microscopy or flow cytometry were based on single cell measurements and data are reported as mean of all cells measured from the same individual (CF patient or healthy control). We found a strong correlation between surface-bound NE activity determined by confocal microscopy and flow cytometry (figure 1f). In addition, these analyses indicated that smFRET flow cytometry detects surface-bound NE activity with less variability and a larger dynamic range (fig. 1e and f). Further, in line with previous results obtained by confocal microscopy [22], forced expiratory volume in $1 \mathrm{~s} \%$ predicted of patients with CF correlated inversely with surface-bound NE activity measured by smFRET flow cytometry (figure 1g).

Taken together, smFRET flow cytometry substantiates previous results obtained by confocal microscopy demonstrating increased surface-bound NE activity on neutrophils sampled from CF airways. In addition, smFRET flow cytometry has several advantages over confocal microscopy. First, smFRET flow cytometry can discriminate between live and dead cells and measurements of NE activity are only based on live sputum neutrophils, thereby overcoming difficulties previously encountered in measurements by confocal microscopy, such as autofluorescence of epithelial cells and interference by extracellular DNA, mucus or dying cells that are excluded from the measurement by flow cytometry. Second, a much larger number of neutrophils can be analysed. Compared to confocal microscopy, where single cell analyses were limited to 100-200 cells, flow cytometry enabled us to increase cell numbers to $\sim 1000$ neutrophils per measurement. This cell number yielded an improved signal-to-noise ratio, but still allowed us to perform paired measurements in sivelestat-treated controls from the same sample, as well as measurements in comparable numbers of neutrophils from CF patients and healthy controls containing substantially lower neutrophil counts. Third, smFRET flow cytometry enables assessment of dynamic changes in surface-bound NE activity over time. Finally, the experimental procedure and data analysis for measurements of NE activity by smFRET flow cytometry is vastly simplified and substantially less time-consuming compared to the confocal microscopy method. In fact, we were able to perform standardised NE activity measurements on neutrophils by flow cytometry within approximately $1 \mathrm{~h}$ (figure $1 \mathrm{~h}$ ), which would be a suitable time for performing this test in the clinical diagnostic setting.

\section{How can this new method for quantitative assessment of elastase activity on} sputum neutrophils be used in the future?

This new method of smFRET flow cytometry enables direct, rapid and dynamic measurement of NE activity on the surface of airway neutrophils (figure $1 \mathrm{~h}$ ). The method can be applied to quantify surface-bound NE activity on neutrophils isolated from sputum of patients with CF and potentially other neutrophilic airway diseases such as bronchiectasis [5]. In addition, data from previous studies in mice with CF-like lung disease using the NEmo-2 reporter and confocal microscopy support the idea that 
measurements can also be performed on cells isolated from BALF [21]. In the clinical arena, we expect smFRET flow cytometry to be used as a versatile translational research tool for more comprehensive studies of the relationship between membrane-bound NE activity and lung disease severity and progression, and studies of its specific value as a biomarker compared to free NE activity, especially at early stages of airway inflammation. In addition, smFRET flow cytometry may also be used as a powerful tool to determine response to therapy to a range of interventions at the level of neutrophil activation and NE secretion. Such interventions may include endogenous and small molecular weight NE inhibitors as a targeted anti-inflammatory strategy [6], as well as emerging highly effective CFTR-directed therapeutics targeting the underlying molecular defect in patients with CF [27, 28]. Furthermore, in combination with cell-specific markers, we envision smFRET flow cytometry as a powerful basic research tool for mechanistic studies of neutrophil pathobiology in the lung, blood and other organs. At a broader scale, this method may also be applied to studies of other proteases on neutrophils and/or other cell types. Beyond NE, lipidated FRET reporters have already been generated for cathepsin G and macrophage elastase (matrix metalloproteinase 12) and are under development for a spectrum of other proteases that have been implicated in CF and other chronic lung diseases [25, 29-31]. With the emergence of these additional reporters, smFRET flow cytometry will also provide new possibilities to obtain a more comprehensive understanding of the dysregulation of protease networks in disease pathogenesis, which may lead to the development of more sensitive biomarkers and novel therapeutic strategies to restore protease-antiprotease balance in CF and other chronic inflammatory lung diseases.

Acknowledgement: We thank Malte Paulsen (Flow Cytometry Core Facility, European Molecular Biology Laboratory, Heidelberg, Germany) for assistance in optimisation of flow cytometry with FRET reporters and Tatjana Uselmann (Thoraxklinik at the University Hospital Heidelberg, Heidelberg, Germany) for assistance in sample collection.

Conflict of interest: M. Hagner has nothing to disclose. D.L. Frey has nothing to disclose. M. Guerra has nothing to disclose. A.S. Dittrich reports grants from German Cystic Fibrosis Association Mukoviszidose e. V. (grant number 1605), during the conduct of the study. V.S. Halls has nothing to disclose. S. Wege has nothing to disclose. F.J.F. Herth reports personal fees from Uptake, BTG, Olympus, Pulmonx and Roche, outside the submitted work. C. Schultz reports grants from Bundesministerium fuer Bildung und Forschung (Deutsches Zentrum fuer Lungenforschung), during the conduct of the study. M.A. Mall reports grants from German Federal Ministry of Education and Research (grant 82DZL004A1), German Research Foundation (grant SFB-TR84TP B08) and Einstein Foundation Berlin (grant EP-2017-393), during the conduct of the study; personal fees for consultancy and lectures from Bayer, personal fees for advisory board work, consultancy and lectures from Boehringer Ingelheim and Vertex Pharmaceuticals, personal fees for advisory board work and consultancy from Polyphor, Arrowhead Pharmaceuticals, ProQR Therapeutics, Spyryx Biosciences, Santhera and Enterprise Therapeutics, personal fees for consultancy from Galapagos and Sterna Biologicals, personal fees for lectures from Celtaxys, outside the submitted work; and is listed on a patent on the Scnnlb-transgenic mouse with royalties paid.

Support statement: Supported in part by grants from the German Ministry for Education and Research (FKZ 82DZL004A1 to M.A. Mall and C. Schultz), the German Cystic Fibrosis Association Mukoviszidose e.V. (Project number 1605 to A.S. Dittrich), the German Research Foundation (SFB-TR84TP B08 to M.A. Mall) and the Einstein Foundation Berlin (EP-2017-393 to M.A. Mall). A.S. Dittrich was supported by a HRCMM (Heidelberg Research Centre for Molecular Medicine) career development fellowship and M. Guerra is a fellow of the Molecular Medicine Partnership Unit (MMPU) between EMBL and the University Clinic Heidelberg. Funding information for this article has been deposited with the Crossref Funder Registry.

\section{References}

1 Sly PD, Brennan S, Gangell C, et al. Lung disease at diagnosis in infants with cystic fibrosis detected by newborn screening. Am J Respir Crit Care Med 2009; 180: 146-152.

2 Sly PD, Gangell CL, Chen L, et al. Risk factors for bronchiectasis in children with cystic fibrosis. N Engl J Med 2013; 368: 1963-1970.

3 Sagel SD, Wagner BD, Anthony MM, et al. Sputum biomarkers of inflammation and lung function decline in children with cystic fibrosis. Am J Respir Crit Care Med 2012; 186: 857-865.

4 Mayer-Hamblett N, Aitken ML, Accurso FJ, et al. Association between pulmonary function and sputum biomarkers in cystic fibrosis. Am J Respir Crit Care Med 2007; 175: 822-828.

5 Chalmers JD, Moffitt KL, Suarez-Cuartin G, et al. Neutrophil elastase activity is associated with exacerbations and lung function decline in bronchiectasis. Am J Respir Crit Care Med 2017; 195: 1384-1393.

6 McKelvey MC, Weldon S, McAuley DF, et al. Targeting proteases in cystic fibrosis lung disease: paradigms, progress, and potential. Am J Respir Crit Care Med 2020; 201: 141-147.

7 Voynow JA, Fischer BM, Malarkey DE, et al. Neutrophil elastase induces mucus cell metaplasia in mouse lung. Am J Physiol Lung Cell Mol Physiol 2004; 287: L1293-L1302.

$8 \quad$ Fahy JV, Dickey BF. Airway mucus function and dysfunction. N Engl J Med 2010; 363: 2233-2247.

9 Fischer BM, Voynow JA. Neutrophil elastase induces MUC5AC gene expression in airway epithelium via a pathway involving reactive oxygen species. Am J Respir Cell Mol Biol 2002; 26: 447-452.

10 Pham CT. Neutrophil serine proteases: specific regulators of inflammation. Nat Rev Immunol 2006; 6: 541-550.

11 Hirche TO, Benabid R, Deslee G, et al. Neutrophil elastase mediates innate host protection against Pseudomonas aeruginosa. J Immunol 2008; 181: 4945-4954.

12 Hartl D, Latzin P, Hordijk P, et al. Cleavage of CXCR1 on neutrophils disables bacterial killing in cystic fibrosis lung disease. Nat Med 2007; 13: 1423-1430. 
13 Weldon S, McNally P, McElvaney NG, et al. Decreased levels of secretory leucoprotease inhibitor in the Pseudomonas-infected cystic fibrosis lung are due to neutrophil elastase degradation. J Immunol 2009; 183: 8148-8156.

14 Margaroli C, Garratt LW, Horati H, et al. Elastase exocytosis by airway neutrophils is associated with early lung damage in children with cystic fibrosis. Am J Respir Crit Care Med 2019; 199: 873-881.

15 Korkmaz B, Attucci S, Juliano MA, et al. Measuring elastase, proteinase 3 and cathepsin G activities at the surface of human neutrophils with fluorescence resonance energy transfer substrates. Nat Protoc 2008; 3: 991-1000.

16 McElvaney OJ, Gunaratnam C, Reeves EP, et al. A specialized method of sputum collection and processing for therapeutic interventions in cystic fibrosis. J Cyst Fibros 2019; 18: 203-211.

17 Owen CA, Campbell MA, Boukedes SS, et al. Cytokines regulate membrane-bound leukocyte elastase on neutrophils: a novel mechanism for effector activity. Am J Physiol 1997; 272: L385-L393.

18 Campbell EJ, Owen CA. The sulfate groups of chondroitin sulfate- and heparan sulfate-containing proteoglycans in neutrophil plasma membranes are novel binding sites for human leukocyte elastase and cathepsin G. J Biol Chem 2007; 282: 14645-14654.

19 Owen CA, Campbell MA, Sannes PL, et al. Cell surface-bound elastase and cathepsin G on human neutrophils: a novel, non-oxidative mechanism by which neutrophils focus and preserve catalytic activity of serine proteinases. J Cell Biol 1995; 131: 775-789.

20 Korkmaz B, Moreau T, Gauthier F. Neutrophil elastase, proteinase 3 and cathepsin G: physicochemical properties, activity and physiopathological functions. Biochimie 2008; 90: 227-242.

21 Gehrig S, Duerr J, Weitnauer M, et al. Lack of neutrophil elastase reduces inflammation, mucus hypersecretion, and emphysema, but not mucus obstruction, in mice with cystic fibrosis-like lung disease. Am J Respir Crit Care Med 2014; 189: 1082-1092.

22 Dittrich AS, Kuhbandner I, Gehrig S, et al. Elastase activity on sputum neutrophils correlates with severity of lung disease in cystic fibrosis. Eur Respir J 2018; 51: 1701910.

23 Gehrig S, Mall MA, Schultz C. Spatially resolved monitoring of neutrophil elastase activity with ratiometric fluorescent reporters. Angew Chem Int Ed Engl 2012; 51: 6258-6261.

$24 \mathrm{Hu}$ HY, Gehrig S, Reither G, et al. FRET-based and other fluorescent proteinase probes. Biotechnol J 2014; 9 : 266-281.

25 Guerra M, Frey D, Hagner M, et al. Cathepsin G activity as a new marker for detecting airway inflammation by microscopy and flow cytometry. ACS Cent Sci 2019; 5: 539-548.

26 Craven TH, Avlonitis N, McDonald N, et al. Super-silent FRET sensor enables live cell imaging and flow cytometric stratification of intracellular serine protease activity in neutrophils. Sci Rep 2018; 8: 13490.

27 Bell SC, Mall MA, Gutierrez H, et al. The future of cystic fibrosis care: a global perspective. Lancet Respir Med 2020; 8: 65-124.

28 Middleton PG, Mall MA, Drevinek P, et al. Elexacaftor-tezacaftor-ivacaftor for cystic fibrosis with a single Phe508del allele. N Engl J Med 2019; 381: 1809-1819.

29 Trojanek JB, Cobos-Correa A, Diemer S, et al. Airway mucus obstruction triggers macrophage activation and matrix metalloproteinase 12-dependent emphysema. Am J Respir Cell Mol Biol 2014; 51: 709-720.

30 Cobos-Correa A, Trojanek JB, Diemer S, et al. Membrane-bound FRET probe visualizes MMP12 activity in pulmonary inflammation. Nat Chem Biol 2009; 5: 628-630.

31 Taggart C, Mall MA, Lalmanach G, et al. Protean proteases: at the cutting edge of lung diseases. Eur Respir J 2017; 49: 1501200 